\section{A) Check for updates}

Cite this: Org. Biomol. Chem., 2019, 17, 1238

Received 13th December 2018,

Accepted 9th January 2019

DOI: $10.1039 / \mathrm{c} 8 \mathrm{ob} 03092 \mathrm{~h}$

rsc.li/obc

\title{
Absolute asymmetric Strecker synthesis in a mixed aqueous medium: reliable access to enantioenriched $\alpha$-aminonitrile $\uparrow$
}

\author{
Shinobu Miyagawa, ${ }^{a}$ Shohei Aiba, ${ }^{a}$ Hajime Kawamoto, ${ }^{a}$ Yuji Tokunaga (D) a and \\ Tsuneomi Kawasaki (iD *b
}

\begin{abstract}
Without using chiral sources, the Strecker reaction of achiral hydrogen cyanide, $p$-tolualdehyde and benzhydrylamine gave enantioenriched L- or D-N-benzhydryl- $\alpha$-( $p$-tolyl)glycine nitriles with up to $>99 \%$ ee in a mixed solvent of water and methanol. Therefore, total spontaneous resolution of $\alpha$-aminonitriles could occur through a prebiotic mechanism of $\alpha$-amino acid synthesis. Moreover, it was demonstrated that the repetition of partial dissolution and crystallization of a suspended conglomerate of aminonitrile under solution-phase racemization could generate the enantiomeric imbalance to afford, in combination with the amplification of chirality, an enantioenriched product in every case. Among the 73 experiments that were carried out, D- and L-enriched isomers occurred 36 and 37 times, respectively. This stochastic behavior, under achiral or racemic starting conditions, meets the requirements of the spontaneous absolute asymmetric Strecker synthesis. The implications of the present results for the origin of chirality of $\alpha$-amino acids are discussed.
\end{abstract}

\section{Introduction}

One of the major interests in science is the origin and amplification of biological homochirality before the emergence of life $;^{1,2}$ that is, the occurrence of an overwhelming predominance of L-amino acids and D-sugars in nature compared with their mirror image molecules. Significant enantiomeric enrichment of biological organic compounds is a signature of life, and can therefore be considered as one of the keys to understanding the origin of life., ${ }^{3,4}$ A main focus of research addresses the question of how the enantiomeric imbalance of the initial chiral compound arose from an achiral or racemic starting environment in the prebiotic world.

Several theories on the origins of chirality have been examined..$^{5-16}$ Among them, spontaneous absolute asymmetric synthesis ${ }^{17}$ has been linked to the generation of enantiomerically enriched compounds. In the homogeneous reaction, asymmetric autocatalysis of 5-pyrimidyl alkanol (Soai reaction $)^{18-20}$ gave the product in nearly enantiomerically pure form without the use of any chiral sources. ${ }^{21-23}$ In the hetero-

${ }^{a}$ Department of Materials Science and Engineering, University of Fukui, Bunkyo, Fukui, 910-8507, Japan

${ }^{b}$ Department of Applied Chemistry, Tokyo University of Science, Kagurazaka, Shinjuku-ku, Tokyo, 162-8601, Japan. E-mail: tkawa@rs.tus.ac.jp

$\dagger$ Electronic supplementary information (ESI) available: Full experimental results. See DOI: 10.1039/c8ob03092h geneous reaction, chiral crystallization including total spontaneous resolution ${ }^{24}$ and stereospecific solid-state reaction have been considered as candidates. ${ }^{13,25-27}$

Asymmetric amplifications, which can enhance an initially induced low enantiomeric excess (ee) to extremely high ee, are essential for the formation of homochirality of organic compounds. ${ }^{28,29}$ Solid-state ee could be generated and amplified by Viedma ripening ${ }^{5,30}$ of a suspended solid under solutionphase racemization, which is a safe method to access enantioenriched chiral organic compounds. ${ }^{31-34}$ In the homogeneous reactions, positive nonlinear effects in asymmetric catalyses ${ }^{35}$ have been observed, including asymmetric autocatalysis. ${ }^{36,37}$ Thus, self-disproportionation of enantiomers is a fundamental phenomenon to improve the enantiopurity of chiral compounds. $^{38}$

Our research focus includes Strecker-type synthesis ${ }^{39-42}$ because this has been considered a possible prebiotic mechanism for $\alpha$-amino acid synthesis since the seminal report on electric discharge experiments conducted under plausible prebiotic conditions. ${ }^{3,43}$ The linkage between the origin of chirality and the formation of prebiotic amino acids may constitute a novel approach that can be used to explain the biological homochirality seen in L-amino acids. Based on this concept, we have reported the spontaneous formation and amplification of enantioenriched $\alpha$-aminonitriles forming conglomerates in methanol, in which the enantiomerically enriched product could be obtained 43 times out of a total of 160 experi- 
(1) Total spontaneous resolution

$$
\begin{gathered}
\begin{array}{c}
\text { Achiral: } \\
\mathrm{HCN}+
\end{array} \\
\text { Amine + Aldehyde } \stackrel{\text { Strecker }}{\stackrel{\text { Preaction }}{\longrightarrow}} \begin{array}{c}
\text { L-or D- } \\
\alpha \text {-aminonitriles }
\end{array} \\
\text { Present work: Solvent }=\mathrm{MeOH} \\
\text { Prelvent }=\mathrm{H}_{2} \mathrm{O}-\mathrm{MeOH}
\end{gathered}
$$

(2) Deracemization of $\alpha$-aminonitrile by temperature cycle

Previous work

$$
\begin{array}{ccc}
\alpha \text {-Aminonitrile } \\
{)} }
\end{array} \stackrel{\text { Amplification of }}{\text { solid ee }} \begin{gathered}
\begin{array}{c}
\text { L- and D- } \\
\alpha-A m i n o n i t r i l e
\end{array} \\
\text { (High ee) }
\end{gathered}
$$

Present work

$\alpha$-Aminonitrile (near-racemic)

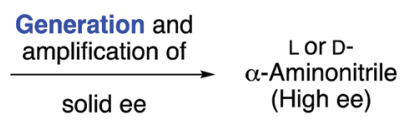

Fig. 1 The concept of the present work.

ments. ${ }^{39}$ The solid-state ee of $\alpha$-aminonitriles could be enhanced significantly ${ }^{39,40}$ by Viedma ripening ${ }^{5,30-33}$ and its modified method, i.e., temperature cycle. ${ }^{34}$

As shown in Fig. 1, the purpose of the present research was to examine (1) the absolute asymmetric Strecker synthesis ${ }^{44}$ (total spontaneous resolution) under aqueous conditions because water is a fundamental solvent in the synthesis of terrestrial prebiotic compounds and (2) deracemization of a near racemic conglomerate of $\alpha$-aminonitrile ${ }^{45}$ by the temperature cycle to generate and amplify the solid-state chirality. These approaches would improve the frequency of the spontaneous generation of enantioenriched aminonitrile.

Here, we demonstrate that the Strecker reaction of three achiral reagents, hydrogen cyanide $(\mathrm{HCN}),{ }^{4}$ amine and aldehyde, in a mixed aqueous medium, gives enantioenriched $\alpha$-aminonitrile with high ee without using any chiral sources. In addition, a previously reported amplification cycle by temperature control ${ }^{40}$ could be used to induce the enantiomeric imbalances in the solid state to provide highly enantiomerically enriched aminonitriles, in which stochastic behavior was observed for the formation frequency of $\mathrm{L}^{-}$and $\mathrm{D}$-enriched compounds.

\section{Results and discussion}

\section{Generation of enantioenriched $\alpha$-aminonitrile as a result of the Strecker reaction in a mixed aqueous medium}

The Strecker reaction between HCN, benzhydrylamine (1), and $p$-tolualdehyde (2) was performed in a 1:1 mixture of water and methanol in the presence of 1,8-diazabicyclo[5.4.0]undec7-ene (DBU) (Fig. 2). ${ }^{46}$ The precipitation of imine $3^{41}$ was observed from the homogeneous reaction mixture, and then the Strecker reaction proceeded gradually to afford $\alpha$-aminonitrile $\mathbf{4}$ as a solid. Imine $\mathbf{3}$ disappeared from the solid phase over three days to give enantioenriched aminonitrile 4 .

As shown in Table 1, L-product $\mathbf{4}$ was isolated as a solid in $65 \%$ yield with $99 \%$ ee (entry 1 ). In contrast, oppositely configured D-solid 4 was obtained in 55\% yield with 53\% ee (entry 2).

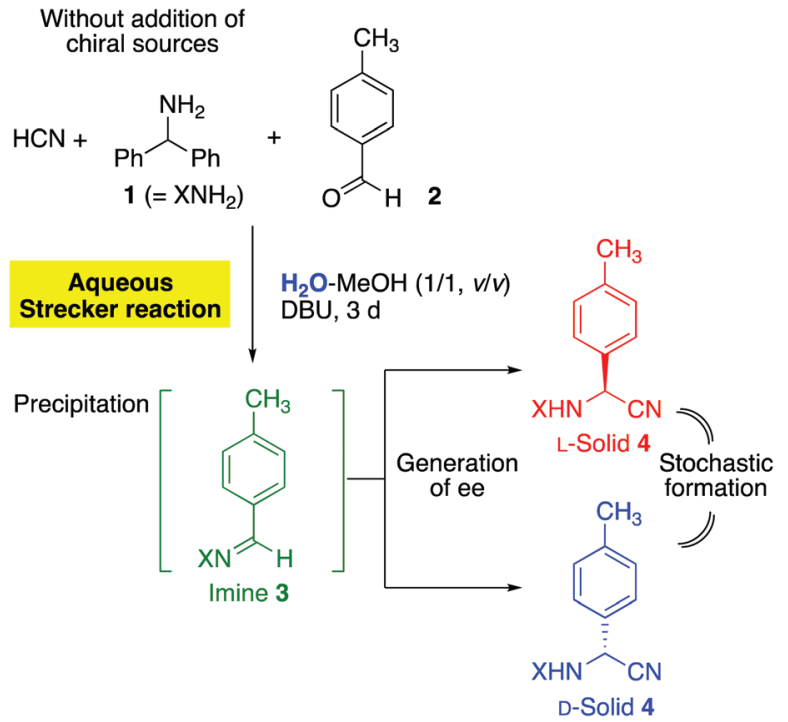

Fig. 2 Spontaneous formation of enantioenriched L- and $D-\alpha$-aminonitrile 4 from the Strecker reaction of three achiral substrates in watermethanol.

Table 1 The absolute handedness and the ee value of $p$-tolylglycine nitrile 4 obtained from the Strecker reaction in the mixed solvent of water and methanol ${ }^{a}$

\begin{tabular}{lll}
\hline & \multicolumn{2}{l}{ Solid $\alpha$-aminonitrile $\mathbf{4}$} \\
\cline { 2 - 3 } Entry & $\mathrm{ee}^{b} / \%$ (config.) & \\
\hline $1^{d}$ & $99(\mathrm{~L})$ & Yield $^{c} \%$ \\
2 & $53(\mathrm{D})$ & 65 \\
3 & $1.0(\mathrm{~L})$ & 55 \\
4 & $99(\mathrm{~L})$ & 62 \\
5 & $99(\mathrm{D})$ & 57 \\
6 & $65(\mathrm{~L})$ & 68 \\
7 & $84(\mathrm{D})$ & 65 \\
8 & $4.9(\mathrm{D})$ & 42 \\
$9-23$ & (Table S1 $\dagger)$ &
\end{tabular}

${ }^{a}$ The reaction was performed in a solution of $1 \mathrm{M} \mathrm{DBU}$ in water/methanol $(1: 1, \mathrm{v} / \mathrm{v})$. The molar ratio used was HCN : $1: 2=1.5: 0.5: 0.5$ $(\mathrm{mmol}){ }^{b}$ The ee value of the solid product, which was determined by high-performance liquid chromatography (HPLC) with a chiral stationary phase. ${ }^{c}$ The isolated yield of the crystalline product by filtration, which was calculated from the theoretical yield based on the molar amount of subjected $\mathbf{1}$ (the same molar amount as 2). The product in the filtrate was not included. ${ }^{d}$ Compound $\mathrm{D}-4$ was isolated in $6 \%$ yield with $12 \%$ ee from the filtrate.

It should be noted that aminonitrile $\mathbf{4}$ can be hydrolyzed under acidic conditions without a decrease in enantiopurity to give $\alpha$-( $p$-tolyl $)$ glycine with the absolute configuration corresponding to that of $\mathbf{4 .}^{39}$

Among the 23 experiments (Table 1, entries 1-23) that were performed, crystallization of $\mathbf{4}$ occurred in every reaction and the distribution of the absolute handedness was approximately stochastic; i.e., $\mathrm{D}-\mathbf{4}$ occurred 11 times and the opposite L-isomer was isolated 12 times. Given that the reaction was initiated by mixing achiral reagents, the present observation 
constitutes one of the conditions necessary for spontaneous absolute asymmetric synthesis. Therefore, in combination with the hydrolysis, enantioenriched $\alpha$-amino acids were obtained through a prebiotic mechanism.

Although the absolute ee values are widely distributed between 1.0 and 99\% ee, a higher frequency of formation of 4 with high ee is observed compared with the previous method. ${ }^{39}$ The critical difference with respect to the present reactions is the precipitation of intermediate imine 3 . It is considered that solid 3 suppresses the formation of 4 in the reaction equilibrium between 3 and $\mathbf{4}$, leading to the gradual formation of solid 4 based on the crystallinities of these compounds. After the formation of the primary nuclei of $\mathrm{D}^{-}$or L-aminonitrile 4 , in principle with high ee, efficient dynamic resolution including amplification by the ripening may proceed to give enantioenriched 4 .

The composition and ee of the suspended solid were assessed by analyzing the samples of the suspended solids (Table S2 $\dagger$ ). After $24 \mathrm{~h}$, more than $95 \%$ of the solid was found to be imine 3. The molar ratio of $3 / 4$ was $38: 62$ and the ee of 4 was $96 \%$ after two days, and finally the solid was composed almost entirely of $\mathbf{4}$ after three days. The final product $\mathbf{4}$ was isolated with $99 \%$ ee by filtration without a decrease in the initially observed $96 \%$ ee. Therefore, highly enantioselective reactive crystallization occurred in conjunction with the Strecker reaction.

\section{Frequent generation of enantioenriched $p$-tolylglycine nitrile 4 by repeated temperature cycles}

The Strecker reaction was then conducted in methanol alone. Under these conditions, the three-component reaction proceeded and the spontaneous crystallization of $\mathbf{4}$ occurred within 1-12 h. Without DBU, the solubility of 4 decreased; thus, 4 precipitated out of every reaction mixture. Even if the initial solid-state ee was below the detectable level, highly efficient absolute asymmetric synthesis can be achieved if solid 4 can be enantioenriched by some method.

As noted, we have reported a significant amplification of the solid-state ee by thermally controlled repetition of partial dissolution and crystallization. ${ }^{40}$ In our simulation, almost the same amount of $\mathrm{L}^{-}$and D-conglomerates 4 (racemate) was dissolved in the heating step; that is, the enantiomeric imbalance was concentrated in the residual solid 4 . Subsequently, 4 was recrystallized at the gradual cooling step to keep the amplified (concentrated) ee of the residual $\mathbf{4}$, as rapid racemization occurred in the solution phase containing DBU. We postulate that this temperature cycle enhances the enantiomeric imbalance, even though the initial imbalance arises from a small fluctuation of the relative amount of enantiomorphs.

The suspension of near-racemic conglomerate $\mathbf{4}$ obtained from methanol was subjected to the cycles after the addition of DBU (Fig. 3A). As shown in Fig. 3B, generation and amplification of solid ee were monitored. In one reaction, an initial generation of L-enrichment $(12 \%$ ee) was observed after three thermal cycles; the ee of $\mathrm{L}-\mathbf{4}$ was subsequently amplified to $26 \%$ ee and to $71 \%$ ee after the fourth and fifth cycles, respect-

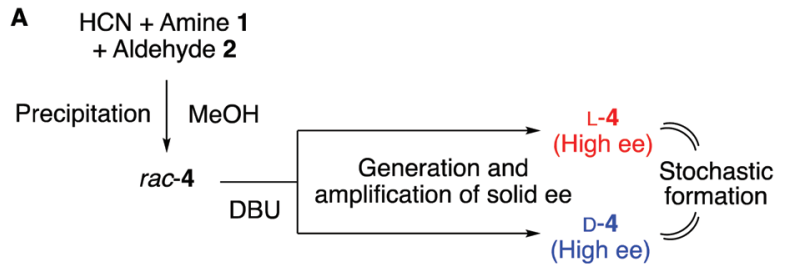

B

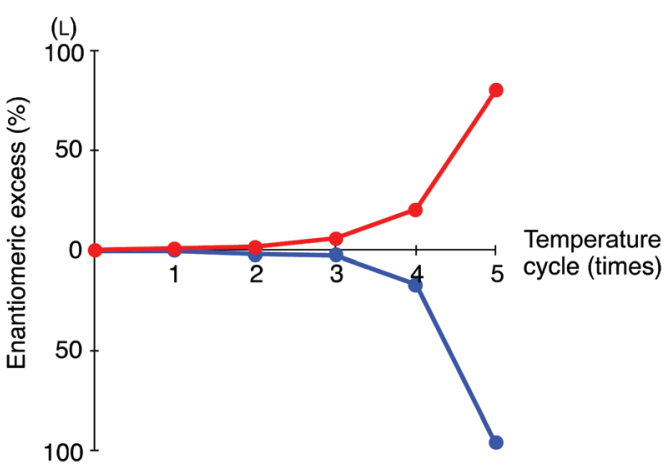

(D)

Fig. 3 Spontaneous generation and amplification of ee in the Strecker reaction in methanol. (a) Schematic outline. (b) Generation and amplification of ee by the temperature cycles (see also Table S3†).

ively (Table 2, entry 1 and Fig. 3B). On the other hand, D-4 was obtained in $40 \%$ yield with $96 \%$ ee after five cycles through the enhancement of the initially observed $2.1 \%$ ee in the second cycle (entry 2 and Fig. 3B). Both $\mathrm{L}^{-}$and $\mathrm{D}-\alpha$-aminonitriles 4 were obtained with 10 and $3.1 \%$ ee values, respectively, after the amplification (entries 3 and 4). Thus, sufficient ee was gen-

Table 2 The absolute handedness and ee of aminonitrile 4 obtained from the Strecker reaction in $\mathrm{MeOH}$ followed by temperature cycles ${ }^{a}$

\begin{tabular}{|c|c|c|c|}
\hline \multirow[b]{2}{*}{ Entry } & \multicolumn{2}{|c|}{ Solid $\alpha$-aminonitrile 4} & \multirow{2}{*}{$\begin{array}{l}\text { Temperature } \\
\text { cycle/times }\end{array}$} \\
\hline & $\mathrm{ee}^{b} / \%$ (config.) & Yield $^{c} / \%$ & \\
\hline $1^{d}$ & $71(\mathrm{~L})$ & 38 & 5 \\
\hline $2^{d}$ & 96 (D) & 40 & 5 \\
\hline 3 & $10(\mathrm{~L})$ & 46 & 4 \\
\hline 4 & 3.1 (D) & 46 & 9 \\
\hline $5^{e}$ & $97(\mathrm{~L})$ & 69 & 11 \\
\hline $6^{e}$ & $93(\mathrm{D})$ & 65 & 11 \\
\hline $7^{f}$ & $88(\mathrm{~L})$ & 65 & 8 \\
\hline $8^{f}$ & $91(\mathrm{~L})$ & 66 & 8 \\
\hline $9^{f}$ & $99(\mathrm{~L})$ & 68 & 12 \\
\hline $10^{f}$ & 99 (D) & 67 & 12 \\
\hline $11-25$ & (Table S4†) & & \\
\hline
\end{tabular}

${ }^{a}$ The molar ratio used was HCN : $1: 2=1.65: 0.45: 0.45(\mathrm{mmol}) .{ }^{b}$ The ee was determined by using HPLC with a chiral stationary phase. ${ }^{c}$ The isolated yield of the crystalline product by filtration (see also Table 1 footnote $c$ ). ${ }^{d}$ Generation and amplification of ee were monitored as shown in Fig. 3B. ${ }^{e}$ After the precipitation of $\mathbf{4}$, the suspension was stirred for $13 \mathrm{~h}$ and concentrated in vacuo. The solid was then completely dissolved in $\mathrm{CHCl}_{3}$ and was separated in portions. After the removal of the solvent, a thermal cycle was applied to the suspension of the resulting solid 4 in $1 \mathrm{M}$ DBU methanol solution, independently. ${ }^{f}$ After the precipitation of $\mathbf{4}$, the suspension was stirred for $15 \mathrm{~h}$, then divided into portions, and then subjected to the procedure given in footnote $e$. The temperature cycle was then conducted independently. 
erated during the cycles, which can be enhanced to high ee. The precipitation of imine $\mathbf{3}$ was not observed under these conditions.

$N$-Benzhydryl- $\alpha$-aminonitriles, formed from the corresponding aldehyde, have a racemization mechanism in only methanol $^{47}$ that can proceed without bases such as DBU. Thus, another interesting issue is whether spontaneously precipitated solid 4 from methanol has an amplifiable solid-state ee. In our previous work, ca. $0.05 \%$ ee could be successfully amplified to $>99.5 \%$ ee. $^{40}$

Near-racemic solid $\mathbf{4}$, obtained from the same reaction, was separated into two portions and these were both subjected to the cycles independently (Table 2, entries 5 and 6). If there was amplifiable ee in the solid, the direction of the $\mathrm{L} / \mathrm{D}$-handedness should be the same after the amplification of each separated sample, we considered. As a result, L- and D-enantiomorphs 4 were obtained from independent cycles. Again, solid 4 was divided into four portions and subsequent amplification gave L-4 in three experimental runs (entries 7-9) and D-4 once (entry 10). Therefore, enough enantioenrichment, which can be amplified by the present method was not generated in the initial precipitation because of a much longer racemization half-life $\left(t_{1 / 2}\right)$ in the solution phase ${ }^{47}$ and rapid crystallization. It should be noted that nearly enantiomerically pure aminonitrile 4 can be synthesized by applying a sufficient number of temperature cycles, as shown in entries 9 and 10 .

To check the distribution of the sense of asymmetry, the sequence of reactions was repeated (Table 2, entries 11-25). Among 25 experiments, D-4 occurred 12 times and L-4 formed 13 times, demonstrating that the absolute handedness seems to be stochastic. In addition, in the absence of chiral sources, an enantioenriched solid product was always obtained by this method. Therefore, repetition of temperature cycles can lead not only to asymmetric amplification but also to spontaneous generation of enantiomeric imbalances; in other words, the process can amplify a stochastic fluctuation of ee in the racemic conglomerate 4 .

Finally, the generation of chirality was then examined starting from solid $\mathbf{4}$ prepared by the homogeneous Strecker reaction (see Scheme in Table 3). It may be conceivable that 4 formed from the homogeneous reaction without the addition of any chiral sources is more racemic than that formed through spontaneous crystallization. Additionally, aminonitrile 4 does not racemize in aprotic solvents such as toluene, ether and $\mathrm{CHCl}_{3}$. Thus, crystallization from these aprotic solvents may give more racemic conglomerates than those obtained through solution-phase racemization. Generation of solid-state chirality was examined starting from the assumed more racemic conglomerate $\mathbf{4}$ than that obtained from the heterogeneous Strecker reaction.

The Strecker reaction of imine $\mathbf{3}$ was performed in homogeneous toluene solution in the presence of a catalytic amount of DBU. ${ }^{48}$ After the completion of the reaction and removal of DBU, 4 was concentrated to form near racemic solid $\mathbf{4}$, which was suspended in $1 \mathrm{M} \mathrm{DBU}$ in methanol and subjected to the temperature cycles.
Table 3 Emergence of enantiomeric imbalances of aminonitrile 4 synthesized by the repetition of heating-cooling of rac-4 obtained from a homogeneous solution without the addition of a chiral material

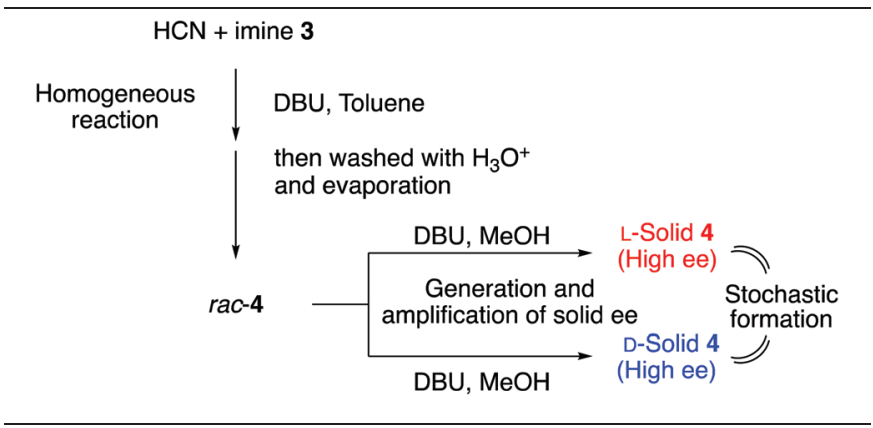

\begin{tabular}{llll}
\hline & \multicolumn{2}{l}{ Solid $\alpha$-aminonitrile $\mathbf{l}$} & \\
\cline { 2 - 3 } Entry & ee $^{a} / \%$ (config.) & Yield $^{b} \%$ & $\begin{array}{l}\text { Temperature } \\
\text { cycle/times }\end{array}$ \\
\hline 1 & $90(\mathrm{D})$ & 49 & 5 \\
2 & $79(\mathrm{~L})$ & 36 & 5 \\
$3^{c}$ & $81(\mathrm{D})$ & 55 & 11 \\
$4^{c}$ & $90(\mathrm{~L})$ & 58 & 14 \\
$5^{d, e}$ & $51(\mathrm{D})$ & 52 & 9 \\
$6^{d, e}$ & $75(\mathrm{D})$ & 49 & 9 \\
$7^{d, e}$ & $65(\mathrm{~L})$ & 49 & 14 \\
$8^{c}$ & $>99.5(\mathrm{~L})$ & 46 & 11
\end{tabular}

${ }^{a}$ The ee was determined by using HPLC with a chiral stationary phase. ${ }^{b}$ The isolated yield of the crystalline product by filtration. ${ }^{c, d}$ The Strecker reaction was performed in the same reaction vessel. After the removal of DBU, the solution was separated in portions and the solvent was removed. The suspension of resulting $\mathbf{4}$ was subjected to temperature cycles, independently. ${ }^{e}$ Before temperature cycle, the homogeneous Strecker reaction was performed in mixed toluene/ methanol solvent $(1: 1, \mathrm{v} / \mathrm{v})$ without DBU.

As shown in Table 3, D-4 was formed with $90 \%$ ee after five cycles (entry 1). In contrast, L-4 was isolated in 36\% yield with $79 \%$ ee (entry 2). Therefore, starting from the racemic conglomerate of aminonitrile 4 obtained from the homogeneous Strecker reaction, the present method induced an ee and give enantioenriched L- or D-4. As shown in entries 3 and 4 , when 4 obtained from the same reaction was divided into two portions, the composition of the resulting 4 became $\mathrm{L}^{-}$and $\mathrm{D}-\mathbf{4}$ with 81 and $90 \%$ ee, respectively. Again, when near rac-4 was divided into three portions (entries 5-7), D-4 occurred twice and $\mathrm{L}-\mathbf{4}$ once. It was possible to enhance the solid-state ee to near enantiopure by applying a sufficient number of cycles (entry 8).

To examine the distribution of the sense of chirality, additional experiments were conducted (Table 3, entries 9-25). Among 25 experiments, D-4 occurred 13 times and L-4 was obtained 12 times. Therefore, the observed stochastic outcomes of the molecular handedness suggest that these conditions enable absolute asymmetric synthesis.

The distributions of absolute configuration and ee of 4 obtained from each method are summarized as histograms in Fig. 4. The absolute configurations seemed to be approximately stochastic under each of the three conditions; namely, the Strecker reaction in the mixed solvent of water and metha- 

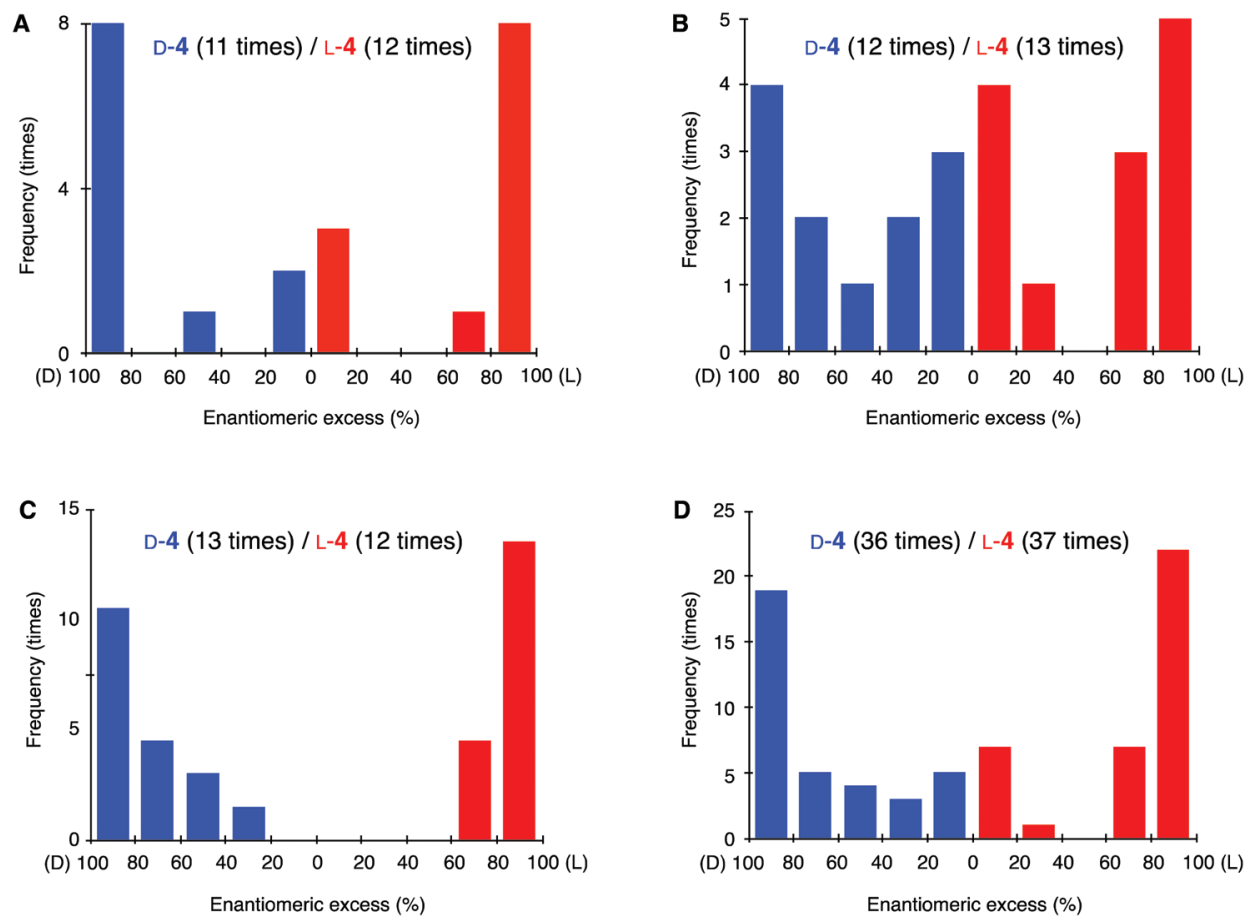

Fig. 4 Histogram summarizing the absolute configuration and enantiomeric excess of aminonitrile 4 (A) by the reaction in the mixed solvent of water and methanol, (B) starting from rac-4 obtained from the heterogeneous reaction in methanol, (C) starting from rac-4 obtained from the homogeneous reaction in toluene and (D) as the total of (A)-(C).

nol (Fig. 4A), heterogeneous reaction (Fig. 4B), and homogeneous reaction (Fig. 4C). The overall experimental results are summarized in Fig. 4D. Among 73 experiments, enantioenriched product 4 could be obtained in every case; enantiomer D-4 occurred in 36 experiments and L-4 in 37 experiments. Thus, the sequence of the reaction seems to proceed without the intervention of external chiral effects. Absolute asymmetric synthesis was thereby realized by the simple control of the temperature of the reaction mixture. Although the distribution of ee values of solid aminonitriles $\mathbf{4}$ were varied between three methods (Fig. 3A-C), it should be noted that the value can be increased to $>99.5 \%$ ee by applying further temperature cycles.

\section{Conclusions}

We have demonstrated that the absolute asymmetric Strecker amino acid synthesis can be conducted under aqueous conditions starting from achiral conditions to afford enantioenriched aminonitriles in the solid state. Even though the reactions started from near-racemic aminonitriles prepared from heterogeneous and homogeneous conditions, the present method induced an initial asymmetry that was enhanced significantly during subsequent amplification. By simple thermal control of the reaction suspension, highly enantioenriched chiral intermediates of $\alpha$-amino acids with either $\mathrm{L}^{-}$or $\mathrm{D}$-configurations could be generated in every case. The observed stochastic behaviour for the formation of $\mathrm{L}^{-}$or D-molecular handedness indicates that the process constitutes one of the conditions necessary for spontaneous absolute asymmetric synthesis. The present results increase the efficiency of the Strecker reactions as a prebiotic mechanism of amino acid synthesis to expand to the asymmetric synthesis of $\alpha$-amino acids that is compatible with a prebiotic environment.

\section{Experimental}

p-Tolualdehyde, benzhydrylamine, DBU, and methanol from commercial sources were used after distillation. Commercial distilled water was directly used for the reaction. Hydrogen cyanide (CAUTION!) was prepared from $\mathrm{H}_{2} \mathrm{SO}_{4}$ and $\mathrm{NaCN}$ in water and isolated by distillation (dried over $\mathrm{CaCl}_{2}$ ).

\section{Absolute asymmetric Strecker reaction (Table 1, entry 1)}

To a solution of amine $1(0.5 \mathrm{mmol}, 87 \mu \mathrm{L})$, aldehyde 2 $(0.5 \mathrm{mmol}, 59 \mu \mathrm{L})$, and DBU $(0.4 \mathrm{~mL})$ in methanol $(1 \mathrm{~mL})$ and water $(1 \mathrm{~mL})$ was added $\mathrm{HCN}(1.5 \mathrm{mmol}, 60 \mu \mathrm{L})$ at room temperature. After gentle stirring for 3 days, the resulting solid was collected by filtration and passed through silica gel using a mixture of ether/hexane $(1: 3, \mathrm{v} / \mathrm{v})$ as an eluent to afford $\mathrm{L}-4$ (0.33 mmol, $102 \mathrm{mg}$, 65\% yield) with 99\% ee. After dilution with ether, the filtrate was washed with $1 \mathrm{M}$ aqueous $\mathrm{HCl}$, dried over $\mathrm{Na}_{2} \mathrm{SO}_{4}$, and concentrated in vacuo. The residue was purified by silica gel column chromatography using ether/hexane $(1: 3, \mathrm{v} / \mathrm{v})$ as an eluent to afford $\mathrm{D}-\mathbf{4}(9.5 \mathrm{mg}$, $0.03 \mathrm{mmol}, 6 \%$ yield) with $12 \%$ ee. 
Strecker reaction in methanol followed by temperature cycles (Table 2, entry 1)

To a solution of amine $1(0.45 \mathrm{mmol}, 78 \mu \mathrm{L})$ and aldehyde 2 $(0.45 \mathrm{mmol}, 53 \mu \mathrm{L})$ in methanol $(1 \mathrm{~mL})$ was added $\mathrm{HCN}$ $(1.65 \mathrm{mmol}, 65 \mu \mathrm{L})$ at room temperature with stirring. After the precipitation of $\mathbf{4}, \mathrm{DBU}(0.2 \mathrm{~mL})$ was added. The suspension was heated at $45-55{ }^{\circ} \mathrm{C}$ to dissolve $c a .90 \%$ of the solid, and then cooled slowly to room temperature over a period of $c a$. $1 \mathrm{~h}$. Partial dissolution by heating and recrystallization by cooling (temperature cycle) were conducted five times. After the filtration of the mixture, the solid was washed with a small amount of methanol to afford L-4 (55 mg, $0.171 \mathrm{mmol}, 38 \%$ yield) with $71 \%$ ee.

\section{Strecker reaction in homogeneous solution followed by temperature cycles (Table 3, entry 1 )}

To a solution of imine $3(2.74 \mathrm{~g}, 9.6 \mathrm{mmol})$ and DBU $(0.072 \mathrm{~mL}, 0.48 \mathrm{mmol})$ in toluene $(30 \mathrm{~mL})$ was added $\mathrm{HCN}$ $(1.2 \mathrm{~mL}, 30.5 \mathrm{mmol})$ at room temperature. After stirring overnight, the reaction was quenched with $1 \mathrm{M}$ aqueous $\mathrm{HCl}$ (15 mL) and the mixture was extracted three times with ether. The organic phase was purified by silica gel column chromatography using ether/hexane $(1: 3, \mathrm{v} / \mathrm{v})$ as an eluent to afford 3 (9.4 mmol, 98\% yield), which was dissolved in $\mathrm{CHCl}_{3}$ and a part of the solution was moved to a vial and concentrated in vacuo to afford solid 4 (173 $\mathrm{mg}, 0.55 \mathrm{mmol})$, which was suspended in methanol $(1 \mathrm{~mL})$. DBU $(0.2 \mathrm{~mL})$ and $\mathrm{HCN}(36 \mu \mathrm{L})$ were then added and temperature cycles were applied five times as described above. After the filtration of the reaction mixture, the resulting solid was washed with a small amount of methanol to afford D-4 (84.2 $\mathrm{mg}, 0.27 \mathrm{mmol}, 49 \%$ yield) with $90 \%$ ee.

\section{Conflicts of interest}

There are no conflicts to declare.

\section{Acknowledgements}

This work was supported by the JSPS KAKENHI Grant Numbers JP16K05692, JP17J06244 and JP17H00312. T. K. thanks the Takeda Science Foundation, S. A. thanks the JSPS Research Fellowships for Young Scientists.

\section{Notes and references}

1 J. F. Bada, Nature, 1995, 374, 594.

2 M. Bolli, R. Micura and A. Eschenmoser, Chem. Biol., 1997, 4, 309-320.

3 S. L. Miller, Science, 1953, 117, 528-529.

4 J. D. Sutherland, Angew. Chem., Int. Ed., 2016, 55, 104-121.

5 C. Viedma, Phys. Rev. Lett., 2005, 94, 065504.

6 Y. Inoue, Chem. Rev., 1992, 92, 741-770.

7 S. Pizzarello and A. L. Weber, Science, 2004, 303, 1151.
8 M. Klussmann, H. Iwamura, S. P. Mathew, D. H. Wells Jr., U. Pandya, A. Armstrong and D. G. Blackmond, Nature, 2006, 441, 621-623.

9 Y. Hayashi, M. Matsuzawa, J. Yamaguchi, S. Yonehara, Y. Matsumoto, M. Shoji, D. Hashizume and H. Koshino, Angew. Chem., Int. Ed., 2006, 45, 4593-4597.

10 R. Breslow and M. S. Levine, Proc. Natl. Acad. Sci. U. S. A., 2006, 103, 12979.

11 U. J. Meierhenrich, L. Nahon, C. Alcaraz, J. H. Bredehöft, S. V. Hoffmann, B. Barbier and A. Brack, Angew. Chem., Int. Ed., 2005, 44, 5630-5634.

12 J. M. Ribo, J. Crusats, F. Sagues, J. Claret and R. Rubires, Science, 2001, 292, 2063-2066.

13 I. Weissbuch and M. Lahav, Chem. Rev., 2011, 111, 32363267.

14 D. K. Kondepudi, R. J. Kaufman and N. Singh, Science, 1990, 250, 975-976.

15 K. Soai, S. Osanai, K. Kadowaki, S. Yonekubo, T. Shibata and I. Sato, J. Am. Chem. Soc., 1999, 121, 11235-11236.

16 T. Kawasaki, M. Sato, S. Ishiguro, T. Saito, Y. Morishita, I. Sato, H. Nishino, Y. Inoue and K. Soai, J. Am. Chem. Soc., 2005, 127, 3274-3275.

17 K. Mislow, Collect. Czech. Chem. Commun., 2003, 68, 849864.

18 K. Soai, T. Shibata, H. Morioka and K. Choji, Nature, 1995, 378, 767-768.

19 K. Soai, T. Kawasaki and A. Matsumoto, Acc. Chem. Res., 2014, 47, 3643-3654.

20 K. Soai, T. Kawasaki and A. Matsumoto, Tetrahedron, 2018, 74, 1973-1990.

21 K. Soai, I. Sato, T. Shibata, S. Komiya, M. Hayashi, Y. Matsueda, H. Imamura, T. Hayase, H. Morioka, H. Tabira, J. Yamamoto and Y. Kowata, Tetrahedron: Asymmetry, 2003, 14, 185-188.

22 T. Kawasaki, K. Suzuki, M. Shimizu, K. Ishikawa and K. Soai, Chirality, 2006, 18, 479-482.

23 K. Suzuki, K. Hatase, D. Nishiyama, T. Kawasaki and K. Soai, J. Syst. Chem., 2010, 1, 5.

24 E. Havinga, Biochim. Biophys. Acta, 1954, 13, 171-174.

25 (a) F. Yagishita, H. Ishikawa, T. Onuki, S. Hachiya, T. Mino and M. Sakamoto, Angew. Chem., Int. Ed., 2012, 51, 1302313025; (b) N. Uemura, S. Toyoda, H. Ishikawa, Y. Yoshida, T. Mino, Y. Kasashima and M. Sakamoto, J. Org. Chem., 2018, 83, 9300-9304.

26 P. M. Björemark, S. Olsson, T. Kokoli and M. Håkansson, Chem. - Eur. J., 2015, 21, 8750-8753.

27 T. Matsuura and H. Koshima, J. Photochem. Photobiol., C, 2005, 6, 7-24.

28 M. M. Green, J.-W. Park, T. Sato, A. Teramoto, S. Lifson, R. L. B. Selinger and J. V. Selinger, Angew. Chem., Int. Ed., 1999, 38, 3138-3154.

29 T. Kawasaki, Y. Matsumura, T. Tsutsumi, K. Suzuki, M. Ito and K. Soai, Science, 2009, 324, 492-495.

30 Z. El-Hachemi, J. Crusats, J. M. Ribó, J. M. McBride and S. V. Verdaguer, Angew. Chem., Int. Ed., 2011, 50, 23592363. 
31 (a) W. L. Noorduin, T. Izumi, A. Millemaggi, M. Leeman, H. Meekes, W. J. P. Van Enckevort, R. M. Kellogg, B. Kaptein, E. Vlieg and D. G. Blackmond, J. Am. Chem. Soc., 2008, 130, 1158-1159; (b) C. Viedma, J. E. Ortiz, T. de Torres, T. Izumi and D. G. Blackmond, J. Am. Chem. Soc., 2008, 130, 15274-15275.

32 W. L. Noorduin, A. A. C. Bode, M. van der Meijden, H. Meekes, A. F. van Etteger, W. J. P. van Enckevort, P. C. M. Christianen, B. Kaptein, R. M. Kellogg, T. Rasing and E. Vlieg, Nat. Chem., 2009, 1, 729-732.

33 R. R. E. Steendam, J. M. M. Verkade, T. J. B. van Benthem, H. Meekes, W. J. P. van Enckevort, J. Raap, F. P. J. T. Rutjes and E. Vlieg, Nat. Commun., 2014, 5, 5543.

34 K. Suwannasang, A. E. Flood, C. Rougeot and G. Coquerel, Cryst. Growth Des., 2013, 13, 3498-3504.

35 T. Satyanarayana, S. Abraham and H. B. Kagan, Angew. Chem., Int. Ed., 2009, 48, 456-494.

36 I. Sato, H. Urabe, S. Ishiguro, T. Shibata and K. Soai, Angew. Chem., Int. Ed., 2003, 42, 315-317.

37 G. Storch and O. Trapp, Nat. Chem., 2017, 9, 179-187.

38 J. Han, O. Kitagawa, A. Wzorek, K. D. Klika and V. A. Soloshonok, Chem. Sci., 2018, 9, 1718-1739.

39 T. Kawasaki, N. Takamatsu, S. Aiba and Y. Tokunaga, Chem. Commun., 2015, 51, 14377-14380.

40 S. Aiba, N. Takamatsu, T. Sasai, Y. Tokunaga and T. Kawasaki, Chem. Commun., 2016, 52, 10834-10837.
41 S. Miyagawa, K. Yoshimura, Y. Yamazaki, N. Takamatsu, T. Kuraishi, S. Aiba, Y. Tokunaga and T. Kawasaki, Angew. Chem., Int. Ed., 2017, 56, 1055-1058.

42 N. Takamatsu, S. Aiba, T. Yamada, Y. Tokunaga and T. Kawasaki, Chem. - Eur. J., 2018, 24, 1304-1310.

43 E. T. Parker, M. Zhou, A. S. Burton, D. P. Glavin, J. P. Dworkin, R. Krishnamurthy, F. M. Fernández and J. L. Bada, Angew. Chem., Int. Ed., 2014, 53, 8132-8136.

44 For the aqueous catalytic asymmetric Strecker reaction, see: S. J. Zuend, M. P. Coughlin, M. P. Lalonde and E. N. Jacobsen, Nature, 2009, 461, 968-970.

45 For a recent example of deracemization of the conglomerate of aminonitriles, see: I. Baglai, M. Leeman, K. Wurst, B. Kaptein, R. M. Kellogg and W. L. Noorduin, Chem. Commun., 2018, 54, 10832-10834.

46 Substrates 1 and 2 could not dissolve completely in the mixed solvent with a higher content ratio of water than $50 \%$.

47 The racemization half-life $\left(t_{1 / 2}\right)$ of 4 was measured to be $c a$. $9 \mathrm{~h}$ in methanol $(0.6 \mathrm{mM})$ and $0.5 \mathrm{~min}$ in $1 \mathrm{M} \mathrm{DBU}$ in methanol (12.5 mM). For $t_{1 / 2}$ of a related compound, see: V. Banphavichit, W. Mansawat, W. Bhanthumnavin and T. Vilaivan, Tetrahedron, 2009, 65, 5849-5854.

48 Without DBU, the Strecker reaction between 3 and HCN in toluene took over days and the reaction stopped without completion. 